\title{
Premature death among people with mental illness
}

This Editorial on a linked paper by Lawrence and colleagues spelt one of these author's names incorrectly in the references (BMJ 2013;346:f2969, doi:10.1136/bmj.f2969). In reference 1, the third author's name should have been spelt as Kisely S, not "Kiseley S."
Cite this as: BMJ 2013;346:f3423

(๑) BMJ Publishing Group Ltd 2013 